ISSN 1991-8631

Original Paper

http://indexmedicus.afro.who.int

\title{
Comparative efficacy of Berenil and Samorin in albino rats experimentally infected with current field isolates of Trypanosoma brucei brucei
}

\author{
Oluremilekun Olufunmilayo AJAYI ${ }^{1}$, Elizabeth Legnen TOSHAK ${ }^{1}$, \\ Olarinde Babatunde OBALOTO ${ }^{2}$, Binta ILIYASU ${ }^{3}$, Augustine Chinyere IGWEH ${ }^{3 *}$, \\ Anthony John DADAH ${ }^{4}$ and Christopher Osagie IDEHEN ${ }^{2}$ \\ ${ }^{I}$ Department of Zoology, University of Jos, P.M.B 2084, Jos, Plateau State, Nigeria. \\ ${ }^{2}$ Animal Trypanosomiasis Division, Nigerian Institute for Trypanosomiasis Research, \\ Vom, Plateau State, Nigeria. \\ ${ }^{3}$ Biochemistry and Chemotherapy Division, Nigerian Institute for Trypanosomiasis, \\ Research, Vom, Plateau State, Nigeria. \\ ${ }^{4}$ Field Operations Division, Nigerian Institute for Trypanosomiasis Research, \\ Vom, Plateau State, Nigeria. \\ ${ }^{*}$ Corresponding author; E-mail: igwehac@yahoo.com, Tel: +2347034518859.
}

\begin{abstract}
The efficacy of two standard veterinary trypanocides, diminazene aceturate (Berenil-therapeutic) and isometamidium chloride (Samorin-prophylactic) was compared in albino rats experimentally infected with current field isolate of Trypanosoma brucei brucei (Federe strain). The study consisted of forty albino rats, divided into 8 groups of five animals each. The negative control was uninfected and untreated (Group 1), whereas the positive control was infected and untreated (Group 2). Other groups were treated intramuscularly with either $0.5 \mathrm{mg} / \mathrm{kg}$ or $3.5 \mathrm{mg} / \mathrm{kg}$ body weight of Samorin or Berenil respectively adopting different protocols. Groups 3 and 4 were treated the same day of infection with Berenil and Samorin respectively (treatment was before infection). Groups 5 and 6 were treated at patency (4 days post infection) with Berenil and Samorin respectively. Groups 7 and 8 were infected before treatment on the same day with Berenil and Samorin respectively, and re-challenged with the T.brucei brucei after four days. The results obtained 60 days post treatment showed that the difference between the efficacies of the two drugs was significant $(\mathrm{P}<0.05)$. Berenil cleared the parasites more from the blood of the albino rats than Samorin. From the recorded values of the parameters (body weight, temperature, packed cell volume and parasitaemic profile), it was concluded that Berenil is a more efficacious trypanocide than Samorin, and is recommended as the drug of choice in the treatment of animal trypanosomiasis.
\end{abstract}

(C) 2013 International Formulae Group. All rights reserved.

Keywords: Laboratory, animal, trypanosomiasis, treatment, trypanocides.

\section{INTRODUCTION}

Trypanosomiases consist of a group of important human and animal diseases caused by parasitic protozoa of the genus Trypanosoma (Barret et al., 2003; Harrington, 2011).

(C) 2013 International Formulae Group. All rights reserved. DOI : http://dx.doi.org/10.4314/ijbcs.v7i4.3 
Animal trypanosomiasis is a major constraint to livestock productivity in the tropics and subtropics and has a significant impact on the life of millions of people globally, mainly in Africa, South America and South East Asia.

In Africa, they assume a continental dimension, since the causative parasites are cyclically transmitted by tsetse flies, which infest some 10 million $\mathrm{km}^{2}$ south of the Sahara (MacLeod et al., 2001; Torr et al., 2007; Matthews, 2011; Jacobs et al., 2011). Livestock production constitutes an important component of Nigerian agricultural development, animal protein and raw materials for agro-allied industries; furthermore, the indirect impact of trypanosomiasis in Nigeria includes decrease in crop production, traction power and reduction in work efficiency of both man and animals (Ovbagbedia et al., 2010). Animal trypanosomiasis has been identified as a major hindrance to the efforts to settle the Fulani nomadic grazers (Abenga et al., 2004).

The repertoire of drugs to fight protozoa diseases such as Malaria, Chagas disease, Leishmaniasis and African trypanomiasis is woefully inadequate (Verlinde et al., 2002; Chatelain and Ioset, 2001; Gonzalez and Cerecetto, 2011). Since it is unlikely that any new and less toxic drug will be developed for this disease in the near future, increasing attention is now being focused on the potential of existing compounds, either alone or in combination chemotherapy for improved efficacy and safety.

The three trypanocides commonly used in the control of animal trypanosomiasis in Africa are diminazene aceturate (Berenil), isometamidium chloride (Samorin) and homidium chloride (Novidium) (Geerts et al., 2001); although a prophylactic approach has been suggested as a better option over treatment of animal trypanosomiasis (Corbel and Henry, 2011). However, in Nigeria, there is indiscriminate use of both drugs (Berenil and Samorin) by herd owners in the treatment of animal trypanosomiasis. Therefore, the aim of this study is to compare the prophylactic and therapeutic efficacy of two commonly used trypanocides, Berenil and Samorin, in albino rats using a current field isolate of Trypanosoma brucei brucei (Federe strain) as the infective test organism.

\section{MATERIALS AND METHODS \\ Experimental rats}

Forty albino rats of both sexes (put in separate cages according to sex) reared at the Animal House of the University of Jos, Nigeria, were used for the study. The animals were maintained at the Nigerian Institute for Trypanosomiasis Research (NITR), Vom in compliance with the Principles of Laboratory Animal Care (National Institute of Health Publication No. 86.23, revised 1985). The bloods of the rats were screened for trypanosomes by wet film preparations to ensure that they were parasite free and uninfected prior to the commencement of the study. The animals were weighed and divided into eight groups of five rats each. Rats in each group were randomly chosen to ensure that rats having the same weight were not placed together in the same experimental group. Rats in each group were clearly marked and numbered, and were placed in separate wire cages. All animals were kept under the same management conditions and fed layers mash, maize offal and given water ad libitum in calibrated bottles. The study was carried out at NITR Vom, Plateau State, Nigeria.

\section{Experimental design}

The animals were divided into eight groups with each group consisting of five animals. Group 1 animals were not infected and not treated (Negative control). Group 2 animals were infected and not treated (Positive control). Group 3 animals were treated with Samorin and inoculated with parasite the same day. Group 4 animals were treated with Berenil and inoculated with parasite the same day. Group 5 were inoculated with parasite and treated with Berenil at patency. Group 6 were inoculated 
with parasite and treated with Samorin at patency. Group 7 were treated with Samorin and inoculated the same day and rechallenged after 4 days. Group 8 were treated with Berenil and inoculated the same day and re-challenged after 4 days.

\section{Inoculation of rats with $T$. brucei brucei}

A current field isolate of $T$.brucei brucei (Federe strain) which has been found to be very virulent in rodents in our laboratory maintained by serial passage in albino rats at the Nigerian Institute for Trypanosomiasis Research (NITR) Vom, Plateau State, Nigeria, was used for the inoculation. Blood was obtained from a donor rat and diluted with normal saline. A drop of the diluted blood was examined under the $\mathrm{x} 10$ and $\mathrm{x} 40$ objectives of the microscope. The procedure was carried out according to Herbert and Lumsden (1976) summarized thus: a wet film was made under a 7 x $22 \mathrm{~mm}$ cover glass. The film was examined under $\mathrm{x} 400$ magnification from a field chosen in which the cells are evenly distributed (the cells should not form more than one layer). The number of Trypanosoma in each field was counted and matched with log figure obtained from a reference table. When single or no Trypanosoma is seen in a selected field, a count is made in 5,10 or 20 fields.

\section{Drug administration}

Berenil and samorin were administered intramuscularly at $3.5 \mathrm{mg} / \mathrm{kg}$ and $0.5 \mathrm{mg} / \mathrm{kg}$ body weight respectively. Aseptic precautions were observed before and after the drugs were administered.

\section{Experimental parameters \\ Body weights}

Body weights of each rat were determined by weighing an empty container and after which the rat was put inside the container and weighed. The actual weight of the rats was obtained by subtracting the weight contained of the empty container from the weight for the rats and the container.

\section{Packed Cell Volume (PCV)}

Packed Cell Volume was estimated by filling heparinized capillary tubes to about $3 / 4$ of their lengths with blood obtained from the pricked tail of the rats. The tubes were sealed at one end with cristasel and placed in a microheamatocrict centrifuge with the sealed ends outermost. The tubes were then centrifuged at 12,000 rpm for 5 minutes. The PCV values were then read with a Hawskey PCV reader as a percentage of packed red blood cells to the total volume of whole blood.

\section{Rectal temperatures}

Rectal temperatures were taken by inserting a clinical thermometer into the rectum of the animals and their temperatures read off.

\section{Examination of experimental rats for parasites}

Wet film preparation of blood obtained from the tail of each rat was examined daily and parasitaemia estimated according to the method of Walker (1968) which is summarized thus: a drop of blood was obtained from the tail-end of each rat by pricking with Lancet. The blood obtained was placed on a clean slide and covered with a cover slip. The preparation was then examined microscopically under $\mathrm{x} 40$ objective for the presence of parasites. The Log Equivalent Value (LEV) of the parasitaemia was determined using the formulae below depending on the nature of the parasitaemia. When there is high parasitaemia, the formula used is:

$\mathrm{LEV}=\log$ (Number of parasites per microscopic field X 1000)

When there is scanty or low parasitaemia, the formula used is:

$$
\mathrm{LEV}=\frac{\log [\text { N P M F }] \times 1000}{\text { N F S B P S }}
$$


where

$\mathrm{N} \quad \mathrm{P} \quad \mathrm{M} \quad \mathrm{F}=$ Number of parasites in microscopic field;

N F S B P S = Number of fields searched before parasites was seen.

Monitoring of parasiteamia daily for

disease progression, therapeutic or

prophylactic assessment or relapse of infection continued for 60 days beyond clearance.

\section{Statistical analysis}

The data was subjected to analysis of variance (ANOVA).

\section{RESULTS}

\section{Clinical signs}

Clinical signs observed in groups 2, 5 and 6 rats included fever, emaciation, anorexia and edema. These signs gradually disappeared from groups 5 and 6 rats following treatment with Berenil and Samorin respectively. One case of mortality was recorded in group 2 rats from day 9 post infection.

\section{Body weight}

Rats in groups 1, 3, 4, 5, 6 and 7 showed an appreciable gain in weight. However, there was a slight decrease in the weight of rats in group 7 by day 7 , but by day 14 , the rats started to gain weight until the end of the fourth week, when the weights of the rats were no longer monitored (Figure 1).

\section{Packed Cell Volume (PCV)}

There was decrease in PCV values of rats in group 2 with increasing patency until all rats died by day 17 post infection. Slight fluctuations were observed in the PCV values of rats in the other experimental groups (Figure 2).

\section{Rectal temperature}

The rectal temperature was elevated in group 2 rats, until all the rats died by day 17 post infection. However, there was a stable mean rectal temperature of $37{ }^{\circ} \mathrm{C}$ in the other groups of rats (Figure 3).

\section{Parasitaemia}

Figure 4 shows the level of parasitaemia in the experimental rats. Preexperimental screening of the rats by wet film preparation technique revealed no trypanosome infection. Trypanosomes were detected in the blood of the infected rats in groups 5 and 6, 3-5 days post infection (prepatent period). Mortality was observed in group 2 rats from day 9 till day 17 when all the rats died. Groups 5 and 6 rats treated at patency with Berenil and Samorin respectively became aparasitaemic 3 days after treatment. Group 3 rats (treated with Samorin and infected with parasite same day) were aparasitaemic all through the experimental period. However, in group 4 rats (treated with Berenil and infected with parasite same day) it was observed that rats number 1, 2 and 5 revealed parasites in their blood stream on day 3 post infection and treatment, but were not-parasitaemic days 4 and 6 post infection and treatment. Rats number 3 and 4 in the same group 4 were not parasitaemic throughout the study. It was observed that groups 7 and 8 animals which were infected and treated with Samorin and Berenil same day respectively and rechallenged with the parasites on day 4 post infection and treatment were aparasitaemic throughout the study. There was a significant difference $(\mathrm{P}<0.05)$ between the levels of parasitaemia in the different groups. 


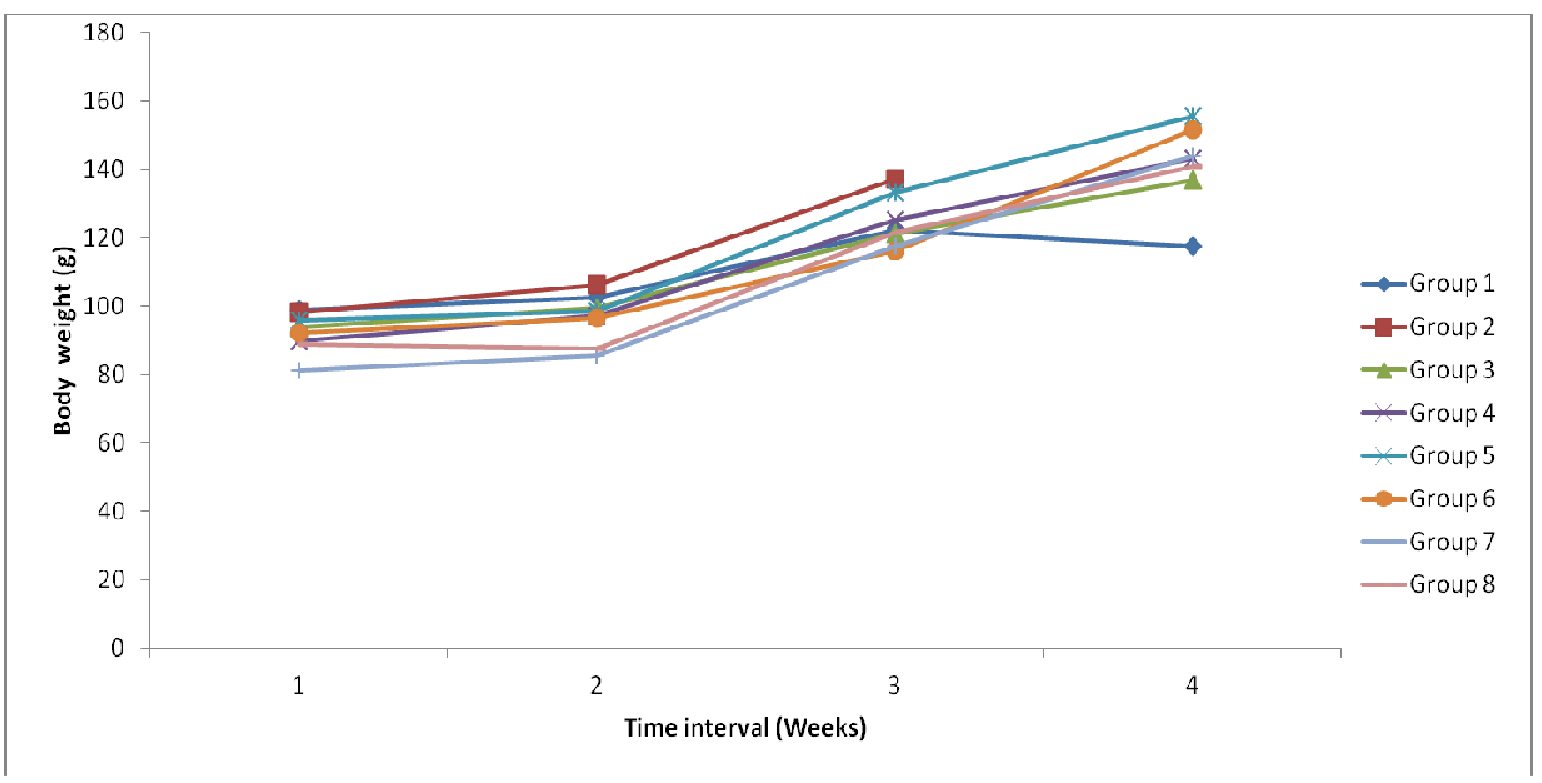

KEY:

Group 1 - Not infected, not treated (Rx) ; Group 2 -Infected, not Rx ; Group 3 -Rx with Samorin, inoculated same day; Group 4 -Rx with Berenil, inoculated same day ; Group 5 -Inoculated with parasite, Rx Berenil at patency; Group 6 -Inoculated with parasite, Rx Samorin at patency ; Group 7 -Rx Samorin, inoculated same day and rechallenged after 4 days ; Group 8 -Rx Berenil, inoculated same day and re-challenged after 4 days;

Figure 1: Body weights of T.brucei infected rats treated with Berenil and Samorin.

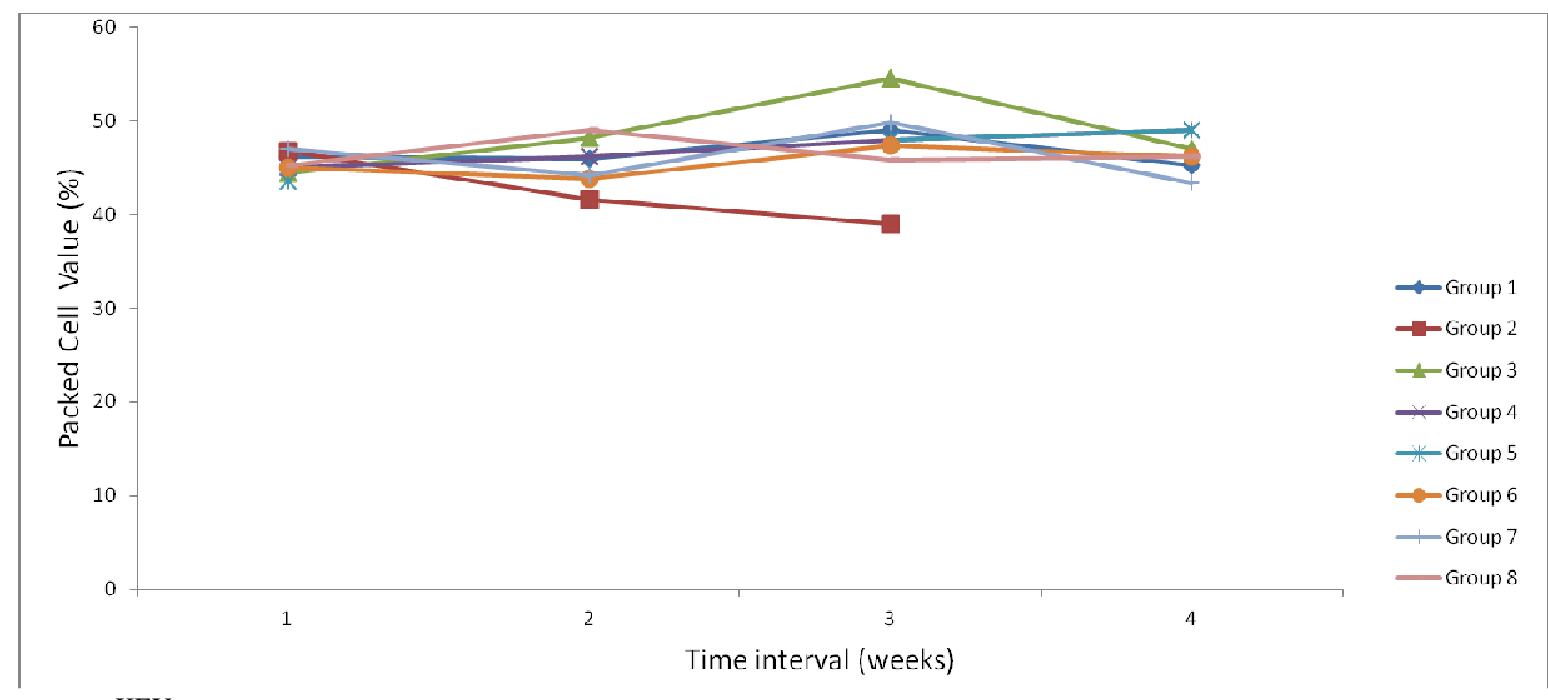

KEY:

Group 1 - Not infected, not treated (Rx); Group 2 -Infected, not Rx ; Group 3 -Rx with Ssmorin, inoculated same day; Group 4 -Rx with Berenil, inoculated same day ; Group 5 -Inoculated with parasite, Rx Berenil at patency ; Group 6 -Inoculated with parasite, Rx Samorin at patency ; Group 7 -Rx Samorin, inoculated same day and rechallenged after 4 days; Group 8 -Rx Berenil, inoculated same day and re-challenged after 4 days

Figure 2: Packed Cell Volume (PCV) of T. brucei infected rats treated with Berenil and Samorin. 


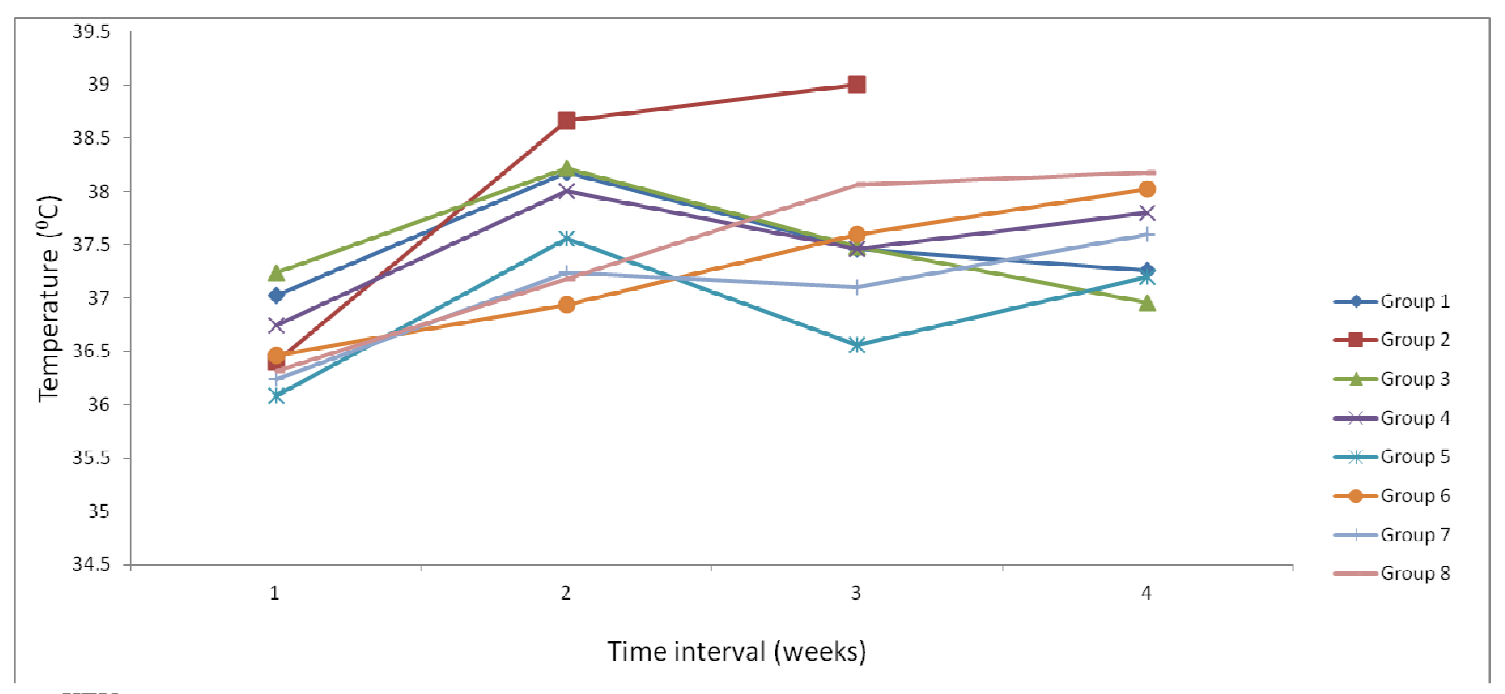

KEY:

Group 1- Not infected, not treated (Rx); Group 2- Infected, not Rx; Group 3- Rx with Samorin, inoculated same day Group 4- Rx with Berenil, inoculated same day; Group 5- Inoculated with parasite, Rx Berenil at patency

Group 6- Inoculated with parasite, Rx Samorin at patency; Group 7- Rx Samorin, inoculated same day and re-challenged after 4 days; Group 8- Rx Berenil, inoculated same day and re-challenged after 4 days

Figure 3: Temperature of T.brucei infected rats treated with Berenil and Samorin.

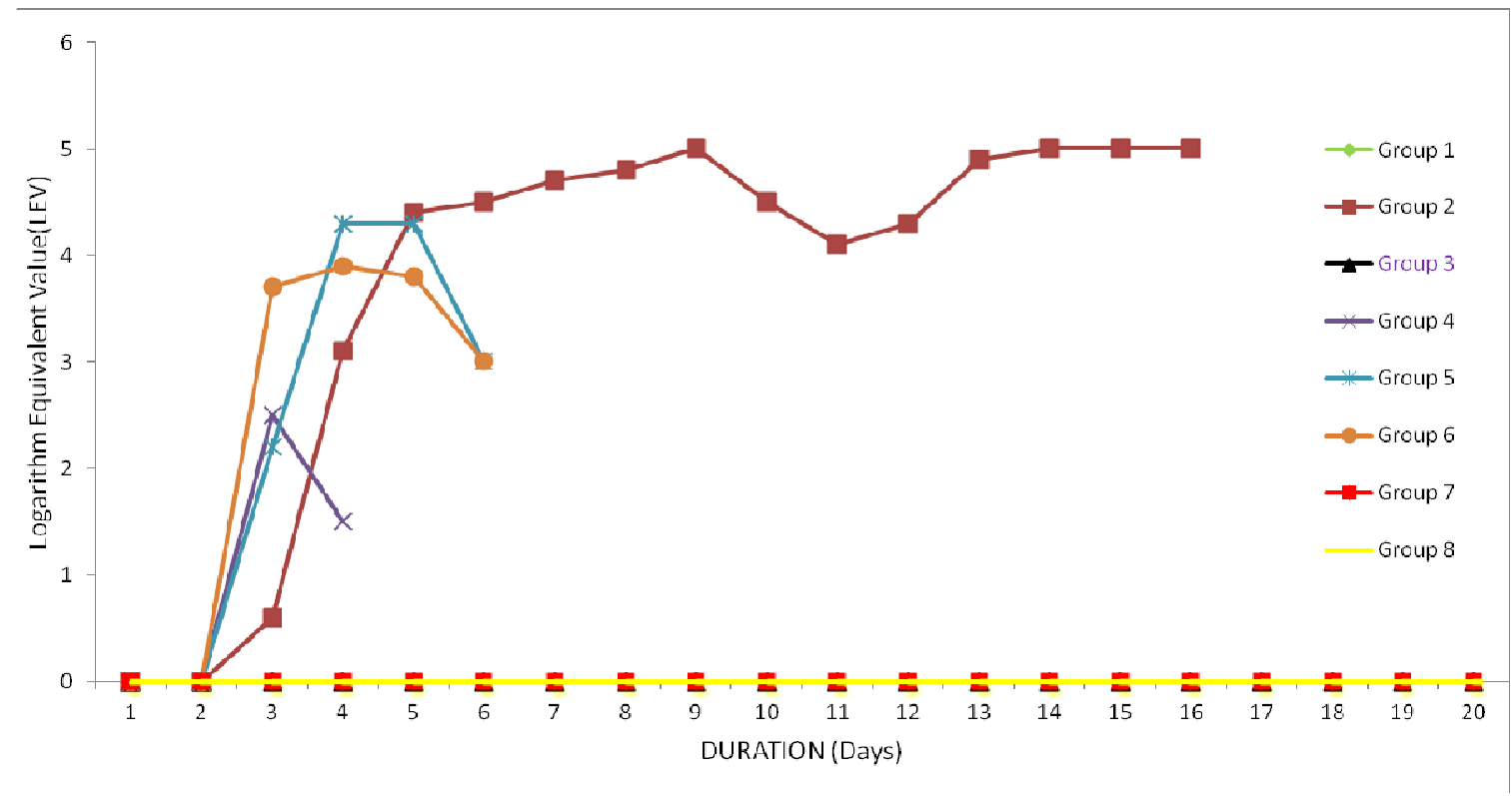

KEY:

Group 1 - Not infected, not treated (Rx); Group 2 -Infected, Not Rx; Group 3 -Rx with Samorin, inoculated same day; Group 4 -Rx with Berenil, inoculated same day ; Group 5 -Inoculated with parasite, Rx Berenil at patency; Group 6 -Inoculated with parasite, Rx Samorin at patency; Group 7 -Rx Samorin, inoculated same day and rechallenged after 4 days; Group 8 -Rx Berenil, inoculated same day and re-challenged after 4 days.

Figure 4: Parasitaemia of T.brucei infected rats treated with Berenil and Samorin. 


\section{DISCUSSION}

The results of the present study show that isometamidium chloride (Samorin) was a more efficacious prophylactic drug than diminazene aceturate (Berenil) even though diminazene which is rapidly excreted has been shown to have a prophylactic protection of 12 days against intravenous challenge with Trypanosoma congolense in cattle. In the present study, rats infected and treated with samorin on the same day developed resistance against infection, whereas rats infected and treated with berenil on the same day were parasitaemic. This is in agreement with the observation of Miruk et al. (2008) that berenil has no prophylactic effect.

The manifestation of parasitaemia in groups 2, 5 and 6 animals indicate that the breed of albino rats used in this study are susceptible to the T.brucei brucei (Federe strain) infection. However, the short prepatent period (3-5days) recorded shows the virulence of the strain (Ezeokonkwo and Agu, 2004).

The experimental increase in the parasitaemia which led to the death of the entire rats in the untreated group within 9-17 days (pi) agrees with the observation of Eghianruwa and Anika (2012) that the survival period of $T$. brucei brucei infection is 1-4 weeks. Aparasitaemia without relapse beyond 60 days in animals treated with either Berenil or Samorin shows the efficacy of the drugs.

Clinical signs like fever, dullness, anorexia observed in rats in the present study are characteristic of animal trypanosomiasis. These observations are in conformity with those of previous workers (Ezeokonkwo and Agu, 2003, 2004; Obidike et al., 2005 and Anene et al., 2006.) Pyrexia which was a common feature in the infected experimental animals is a recognized clinical sign of trypanosomiasis in animals (Dargantes et al., 2005). Pyrexia is due to the accumulation of tryptophan metabolite (tryptophol) in trypanosome infections. Accumulation of tryptophol in pharmacological doses in animals has been reported by Ezeokonkwo et al., (2007) to be responsible for rectal temperature changes or feverish conditions and in the humoral antibody response to heterologous antigen. Treatment with Berenil and Samorin reversed these changes.

There was no significant change $(\mathrm{P}>0.05)$ in the body weights of the experimental rats. This may be attributed to the acute nature of the infection as reported in the present study and in dogs by Ezeokonkwo et al. (2008). Infection with T. brucei brucei had no adverse effect on the PCV values of the animals treated with Samorin and Berenil. However, untreated control (Group2) recorded fall in PCV values with concomitant increase in level of parasitaemia. This fall implies anaemic conditions in the animals. This is in conformity with the findings of Ezeokonkwo and Agu (2003); Obidike et al. (2005); Anene et al. (2006); Behnke et al. (2011). Anaemia in trypanosomiasis has been attributed to a combination of factors, which include haemolysis by trypanosome, haemodilution and erythrophagocytosis (Taylor and Authie, 2004).

\section{Conclusion}

The results of the present study strengthen the use of Samorin as a more efficacious prophylactic drug than Berenil which is more therapeutic. In conclusion, the indiscriminate use of these drugs by Fulani herdsmen in Nigeria in the treatment of animal trypanosomiasis should be discouraged through awareness creation by public enlightenment by the appropriate authorities.

\section{REFERENCES}

Abenga JN, Enwezor FNC, Lawani FAG, Osue HO, Ikemereh, ECD. 2004. Trypanosome prevalence in cattle in Lere in Kaduna State, North Central Nigeria. Rev. d'Elev. Med. Pays Trop., 57(1-2): 45 -48 .

Anene BM, Ezeokonkwo RC, Mmesirionye TI, Tettey JNA, Brock JM, Barret MP, Dekoning HP. 2006. A diminazene resistant strain of Trypanosoma brucei brucei isolated from a dog is cross resistant to pentamidine in experimentally 
infected albino rats. Parasitol., 132: 127133.

Barret MP, Burchmore RJS, Stich A, Lassan JO, Frash AC, Cazzulo J, Krishna S. 2003. The trypanosomiasis. Lancet; 362(394): 1469-1480.

Behnke JM, Chiejina SN, Musongong GA, Nnadi PA, Ngongeh LA, Abonyi FO, Fakae B. 2011. Resistance and resilience of traditionally managed West African Dwarf goats from the savannah zone of northern Nigeria to naturally acquired trypanosome and gastrointestinal nematode infections. J. Helmth., 85(1): 80-91.

Chatelain E, Ioset JR. 2011. Drug development for neglected diseases: the DNDi model. Drug Des Devel Ther., 5: 175-181.

Corbell V, Henry MC. 2011. Prevention and control of malaria and sleeping sickness in Africa: where are we and where are we going? Para. and Vect., 4: 37.

Dargantes AP, Reid SA, Copeman DB. 2005. Experimental Trypanosoma evansi infction in the goat. 1. Clinical signs and clinical patholology. J. Comp. Pathol., 133(4): 261-266.

Eghianruwa KI, Anika SM. 2012. Effects of DMSO on Diminazine efficacy in experimental T.brucei infection. Int. J. Ani. Vet. Adv., 4(2): 2041-2908.

Ezeokonkwo RC, Agu WE. 2003. Experimental infection of rabbits with Trypanosoma brucei alone and mixed infection of Trypanosoma brucei and Trypanosoma congolense: A comparative study. Proceedings of the $28^{\text {th }}$ Annual Conference of the Nigerian Society of Animal Production, held at the Institute of Agricultural Research and Training (IAR \& T) Moor Plantation, Ibadan, March $16^{\text {th }}-20^{\text {th }}$, pp $17-21$.

Ezeokonkwo RC, Agu WE. 2004. Experimental infections of domestic rabbits (Oryctolagus cuniculus) with Trypanosoma brucei and Trypanosoma congolense. A comparative study. Nig .J. Animal Prod., 31(1): 100 -111.
Ezeokonkwo RC, Okoro FC, Ezeh IO. 2007. The efficacy of increasing doses of Samorenil $^{\mathrm{R}}$ in the treatment of Trypanosoma brucei infected albino rats. Nig. Vet. J., 28(2): 24-32.

Ezeokonkwo RC, Akpa PO, Eze CA. 2008. Comparative efficacy assessment of pentamidine isothionate and diminazene aceturate in the chemotherapy of Trypanosoma brucei brucei infection in dogs. Vet. Parasitol., 151:139 - 149.

Geerts S, Holmes PH, Eisler MC, Diall O. 2001. African bovine trypanosomiasis: The problem of drug resistance. Parasitol. Tod., 17(1): 25-28.

Gonzalez M, Cerecetto H. 2011.Novel compounds to combat trypanosomatid infections: a medicinal chemical perspective. Experts Opinions on Thera. Pat., 21(5): 699-715.

Harrington JM. 2011. Antimicrobial peptide killing of African trypanosomiasis. Parasite Immunol., 33(8): 461-469.

Jacobs RT, Nare B, Phlips MA. 2011. State of the art in African drug discovery. Current Topics Med.Chem., 11(10) : 1255-1274.

MacLeod A, Tait A, Turner CMR. 2001. The population genetics of Trypanosoma brucei and the origin of infectivity. Phil. Trans. The Roy. Soc. Lon., Series BBiololgical Sciences, 356(1411): 10351044.

Matthews KR. 2011. Controlling and coordinating development in vector transmitted parasites. Sci., 331(6021): 1149-1153.

Miruk A, Hagos A, Yacob HT Asnake F, Basu AK. 2008. Prevalence of bovine trypanosomiasis and trypanocidal drug sensitivity studies of Trypanosoma congolense in Wolyta and Dawero zones of Southern Ethopia. Vet. Paraitol., 152(1-2): 141-147.

Obidike IR, Aka L, Momah CV, Ezeokonkwo RC. 2005. Effects of Trypanosoma brucei brucei infections in diminazene aceturate treatment on serum activities of certain enzymes. Sah. J. Vet. Sci., 4(1): 17-23. 
Ovbagbedia PR, Samdi, SM, Wayo B, Bizi R. 2010. Impact of trypanosomosis on food security in Nigeria: a review. $34^{\text {th }}$ Annual Conference of Parasitology and Public Health Society of Nigeria, held at the Federal University of Technology Owerri, Imo State. September 21-25, 2010. Abstract No. 91.

Taylor K, Authie EM.-L. 2004. Pathogenesis of animal trypanosomiasis. In The Trypanosomiasis, Maudlin I, Holmes PH, Miles MA (eds). CABI Publishing: Wallingford UK; 331-353.

Torr SJ, Prior A, Wilson PJ, Schofield S. 2007. Is there safety in number? The effect of cattle herding on biting risk from tsetse flies. Med. Vet. Ento., 21(4): 301311.

Verlinde CIMJ, Bressi JC, Choe J, Suresh S, Buckner FS, Van Voorhis WC, Michels, PAM, Gelb, MH, Hol WCJ. 2002. Protein structure-based design of anti-protozoal drugs. J. Braz. Chem. Soc., 13(6): 843848.

Walker PJ. 1968. Standardization of routine observation of blood for trypanosomes. EATRO Report, pp 15-16. 